\title{
Diffusion processes and the asymptotic bulk gap probability for the real Ginibre ensemble
}

\author{
Peter J. Forrester
}

School of Mathematics and Statistics, ARC Centre of Excellence for Mathematical \& Statistical Frontiers, The University of Melbourne, Victoria 3010, Australia; email: p.forrester@ms.unimelb.edu.au

\begin{abstract}
It is known that the bulk scaling limit of the real eigenvalues for the real Ginibre ensemble is equal in distribution to the rescaled $t \rightarrow \infty$ limit of the annihilation process $A+A \rightarrow \emptyset$. Furthermore, deleting each particle at random in the rescaled $t \rightarrow \infty$ limit of the coalescence process $A+A \rightarrow A$, a process equal in distribution to the annihilation process results. We use these inter-relationships to deduce from the existing literature the asymptotic small and large distance form of the gap probability for the real Ginibre ensemble. In particular, the leading form of the latter is shown to be equal to $\exp (-(\zeta(3 / 2) /(2 \sqrt{2 \pi})) s)$, where $s$ denotes the gap size and $\zeta(z)$ denotes the Riemann zeta function. It is shown how this can be rigorously established using an asymptotic formula for matrix Fredholm operators. A determinant formula is derived for the gap probability in the finite $N$ case, and this is used to illustrate the asymptotic formulas against numerical computations.
\end{abstract}

Dedicated to Professor R.J. Baxter on the occasion of his $75^{\text {th }}$ birthday.

\section{Introduction}

For a point process on the line, a natural statistical quantity is the probability distribution for consecutive spacings between points. As an example, suppose the point process is a perfect gas in equilibrium with density $\rho$. Then the probability density function for the distribution of consecutive spacings, $p_{0}(s)$ say, is given by the Poisson distribution $p_{0}(s)=\rho e^{-\rho s}, s \geq 0$. A feature of the Poisson distribution is that it is a maximum for zero separation, telling us that particles in a perfect gas tend to clump, while there is exponential decay at large separation.

Our interest is in $p_{0}(s)$ for point process defined by the real eigenvalues of real standard Gaussian random matrices (matrices from the real Ginibre ensemble [21], to be abbreviated $\mathrm{rG}$ below). The interest in $p_{0}(s)$ for eigenvalues of random matrices goes back to Wigner 
in the 1950's (see [30] and references therein). The problem being addressed was that of providing a theoretical explanation for the statistical properties of highly excited states of certain heavy nuclei, in sectors specified by definite quantum numbers. A significant property of the energy levels is that they can be unfolded, which means scaled by the mean spacing to get parameter independent probability distributions, and in particular a parameter independent form of $p_{0}(s)$. In relation to the latter Wigner hypothesized the functional form

$$
p_{0}^{\mathrm{W}}(s)=\frac{\pi}{2} s e^{-\pi s^{2} / 4},
$$

now known as the Wigner surmise. Note that (1.1) differs both in its small and large distance form to that of the Poisson distribution. Specifically, the linear decay as $s \rightarrow 0$ quantifies the observed effect of repulsion between consecutive energy levels in experimental data, while the exponential large $s$ form of the Poisson distribution is replaced by a Gaussian.

In the course of this study Wigner introduced ensembles of real symmetric random matrices - referred to as the Gaussian orthogonal ensemble (GOE); see e.g. [14, as a theoretical model underlying (1.1). Specifically, Wigner argued that the scaled bulk eigenvalues of such matrices would have the same statistical distributions as those for the unfolded large energy levels of the complex nuclei. The task then was to compute $p_{0}(s)$ for the bulk scaling limit of the GOE. In a major achievement, this was carried out by Gaudin [20], who first expressed $p_{0}(s)$ in terms of a Fredholm determinant, and then gave a computable expression for the latter in terms of prolate spherical functions. (As an aside we remark that it is now realised that the Fredholm form determinant itself is well suited to direct numerical computation [6, 7].) This work demonstrated that (1.1) is not exact, but nonetheless differs by at most a few percent in relative accuracy. Later, building on the work [24], it was found [19] that the exact form of $p_{0}(s)$ in fact permits the general structure of (1.1),

$$
p_{0}(s)=\frac{2 u\left((\pi s / 2)^{2}\right)}{s} \exp \left(-\int_{0}^{(\pi s / 2)^{2}} \frac{u(t)}{t} d t\right)
$$

(11.1) corresponds to the choice $u(t)=t / \pi$ ), where $u$ is a particular solution of a certain $\sigma$ form Painlevé V nonlinear differential equation. The exact result (1.2) exhibits the small distance expansion

$$
p_{0}(s)=\frac{\pi^{2} s}{6}-\frac{\pi^{4} s^{3}}{60}+\frac{\pi^{4} s^{4}}{270}+O\left(s^{5}\right)
$$

while for $s \rightarrow \infty$

$$
p_{0}(s)=e^{-(\pi s)^{2} / 16-\pi s / 4+(17 / 8) \log s+O(1)}
$$

(see e.g. [18] and references therein). Thus the small distance linear repulsion of (1.1), and its large distance Gaussian decay are conserved by the exact behaviours, although in both cases with different proportionality constants.

Our interest in this paper is to derive the analogue of the expansions (1.3) and (1.4) for the point process defined by bulk real eigenvalues of the real Ginibre ensemble. For 
large matrix size $N$, it is known that the expected number of real eigenvalues is to leading order equal to $\sqrt{2 N / \pi}$, and the bulk density is equal to $1 / \sqrt{2 \pi}$ [12]. This point process shares with the GOE eigenvalues the special feature that it is an example of a Pfaffian point process. Thus the $k$-point correlation function can be expressed as a $2 k \times 2 k$ Pfaffian with matrix elements independent of $k$. In the bulk scaling limit this reads [16, 35, 8]

$$
\rho_{(n)}^{\mathrm{rG}}\left(\lambda_{1}, \ldots, \lambda_{n}\right)=\operatorname{Pf}\left[\begin{array}{ll}
-I\left(\lambda_{i}, \lambda_{j}\right) & S\left(\lambda_{i}, \lambda_{j}\right) \\
-S\left(\lambda_{j}, \lambda_{i}\right) & D\left(\lambda_{i}, \lambda_{j}\right)
\end{array}\right]
$$

where

$$
\begin{aligned}
S(x, y) & =\frac{1}{\sqrt{2 \pi}} e^{-(x-y)^{2} / 2} \\
I(x, y) & =\frac{1}{2} \operatorname{sgn}(y-x)-\int_{x}^{y} S(x, u) d u \\
D(x, y) & =\frac{\partial}{\partial x} S(x, y) .
\end{aligned}
$$

Recently it has been proved that (1.5) and (1.6) hold for the real eigenvalues of a much larger class of non-Hermitian random matrices with real entries than just the real Ginibre ensemble [36].

In general the $k$-point correlation functions can be used to compute spacing distributions. Thus let $E(k ; J)$ denote the probability that the interval $J$ contains exactly $k$ eigenvalues (in particular, $E(0 ;(0, s))$ is referred to as the gap probability), and define the corresponding generating function by

$$
E(J ; \xi)=\sum_{k=0}^{\infty}(1-\xi)^{k} E(k ; J)
$$

Then we have

$$
E(J ; \xi)=1+\sum_{n=1}^{\infty} \frac{(-\xi)^{n}}{n !} \int_{J} d x_{1} \cdots \int_{J} d x_{n} \rho_{(n)}\left(x_{1}, \ldots, x_{n}\right) .
$$

It is of interest to remark that $E(J ; \xi)$, for $0<\xi \leq 1$, also has the interpretation of there being no eigenvalues (and thus as a gap probability) in the interval $J$ of the original system diluted so that each eigenvalue is independently deleted with probability $(1-\xi)$ (see e.g. [14, paragraph below (9.4)]). In the special case that $\rho_{(n)}$ has the Pfaffian structure (1.5), it is generally true that (1.7) can be summed to give a Fredholm determinant. In this regards, let $Z_{2}:=\left[\begin{array}{cc}0 & -1 \\ 1 & 0\end{array}\right]$, and transform the $2 \times 2$ blocks in (1.5) by multiplication on the right by $Z_{2}$ to thus obtain

$$
\left[\begin{array}{ll}
S\left(\lambda_{i}, \lambda_{j}\right) & I\left(\lambda_{i}, \lambda_{j}\right) \\
D\left(\lambda_{i}, \lambda_{j}\right) & S\left(\lambda_{j}, \lambda_{i}\right)
\end{array}\right]=: K\left(\lambda_{i}, \lambda_{j}\right)
$$


Furthermore, let $K_{J}$ denote the corresponding $2 \times 2$ matrix integral operator with kernel $K(x, y)$ supported on $J$. Then we know from [14, eq. (9.181)] that (1.5) and (1.7) imply

$$
\left(E^{\mathrm{rG}}(J ; \xi)\right)^{2}=\operatorname{det}\left(\mathbb{I}_{2}-\xi K_{J}\right) .
$$

Since

$$
p_{0}(s)=\frac{1}{\rho} \frac{d^{2}}{d s^{2}} E((0, s) ; \xi=1),
$$

to obtain the analogues of (1.3) and (1.4), it suffices to deduce the corresponding expansions of the Fredholm determinant in (1.9). In fact the sought expansions - but not derived from (1.9) - are already in the literature, and reveal unobvious relations with other point processes, as we will now proceed to detail.

\section{Relationship to diffusion processes}

\subsection{The coalescence process $A+A \rightarrow A$}

In recent years attention has focussed on (1.1), and its analogue for the $k$-th nearest neighbour spacings distributions, as an ansatz for the spacing distribution in certain onedimensional non-equilibrium statistical mechanical models [22, 23]. In fact, as will now be revised, (1.1) is the exact result for the $t \rightarrow \infty$ rescaled consecutive spacing distribution in diffusion limited coalescence $A+A \rightarrow A$.

Let us first define the latter. Starting from a point distribution on the real line with uniform density $\rho$, each particle performs independent Brownian motion. When two particles meet they immediately merge into one. The dynamical particle density $\rho(x ; t)$ therefore decreases with time. One has $\rho(x ; t) \propto t^{-1 / 2}$ [5. Eq. (16)], and in particular the system is not in equilibrium. Nonetheless, by rescaling the particle positions so that the density is again uniform and independent of time, the $t \rightarrow \infty$ steady state has some special properties which we will now revise.

Specifically, consider the gap probability $E(0 ; J), J=\left\{\left(x_{2 i-1}, x_{2 i}\right)\right\}_{i=1, \ldots, n}$, with

$$
x_{1}<x_{2}<\cdots<x_{2 n-1}<x_{2 n} .
$$

Then as $t \rightarrow \infty$, and with the density rescaled to a constant value $\rho$, one has the exact result [5, Eq. (11) with eq. (15) substituted]

$$
E^{\mathrm{c}}(0 ; J)=\operatorname{Pf} A
$$

where $A$ is the antisymmetric matrix with entries $(i j), i<j$,

$$
A_{i j}=\operatorname{erfc}\left(\frac{\sqrt{\pi} \rho}{2}\left(x_{j}-x_{i}\right)\right)
$$


(the superscript $c$ on $E^{\mathrm{c}}$ refers to the coalescence process). One recalls that a Pfaffian of an even degree antisymmetric matrix $A=\left[\alpha_{j, k}\right]_{j, k=1, \ldots, 2 n}, \alpha_{j, k}=-\alpha_{k, j}$, is by definition (see e.g. [14, Def. 6.1.4])

$$
\operatorname{Pf} A=\sum_{P \in S_{2 n}: P(2 l)>P(2 l-1)} \varepsilon(P) \alpha_{P(1), P(2)} \alpha_{P(3), P(4)} \cdots \alpha_{P(2 n-1), P(2 n)},
$$

where $S_{2 n}$ is the set of all permutations of $\{1,2, \ldots, 2 n\}$, the restriction $P(2 l)>P(2 l-1)$ is required for each $l=1, \ldots, n$, and furthermore the sum is restricted to distinct terms only. In [5] the expression for (2.2) is the summation corresponding to the RHS of (2.5) only, and in particular the fact that this is a Pfaffian is not mentioned. In the simplest case of $J=(0, s),(2.2)$ and (2.3) give

$$
E^{\mathrm{c}}(0 ;(0, s))=\operatorname{erfc}\left(\frac{\sqrt{\pi} \rho}{2} s\right)
$$

and application of (1.10) implies

$$
p_{0}^{\mathrm{c}}(s)=\frac{\rho \pi s}{2} e^{-\pi(\rho s)^{2} / 4},
$$

thus realizing the Wigner surmise (1.1).

One significance of (2.2), (2.3) is that the exact form of the $n$-point correlation then follows from the general formula

$$
\rho_{(n)}\left(y_{1}, \ldots, y_{n}\right)=(-1)^{n} \frac{\partial^{n}}{\partial x_{2} \partial x_{4} \ldots \partial x_{2 n}} E\left(0 ;\left(x_{1}, x_{2}\right), \ldots,\left(x_{2 n-1}, x_{2 n}\right)\right),
$$

with the RHS evaluated at $x_{2 i}=x_{2 i-1}=y_{i},(i=1, \ldots, n)$. Specifically, we read off from [27, eq. (20)] that

$$
\rho_{(n)}\left(y_{1}, \ldots, y_{n}\right)=(-\rho)^{n} \operatorname{Pf} \tilde{A},
$$

where $\tilde{A}$ is the anti-symmetric matrix with upper triangular entries

$$
\begin{aligned}
\tilde{A}_{2 i-1,2 j-1}=\operatorname{erfc}\left(\frac{\sqrt{\pi} \rho}{2}\left(y_{j}-y_{i}\right)\right), & \tilde{A}_{2 i-1,2 j}=-e^{-\pi \rho^{2}\left(y_{j}-y_{i}\right)^{2} / 4} \\
\tilde{A}_{2 i, 2 j-1}=e^{-\pi \rho^{2}\left(y_{j}-y_{i}\right)^{2} / 4}, & \tilde{A}_{2 i, 2 j}=-\frac{\pi \rho}{2}\left(y_{j}-y_{i}\right) e^{-\pi \rho^{2}\left(y_{j}-y_{i}\right)^{2} / 4} .
\end{aligned}
$$

This assumes the ordering $y_{1}<\cdots<y_{n}$. However, noting that for $y_{j}>y_{i}$

$$
\operatorname{erfc}\left(\frac{\sqrt{\pi} \rho}{2}\left(y_{j}-y_{i}\right)\right)=\operatorname{sgn}\left(y_{j}-y_{i}\right)-\frac{2}{\sqrt{\pi}} \int_{0}^{\sqrt{\pi} \rho\left(y_{j}-y_{i}\right) / 2} e^{-t^{2}} d t
$$

we see that $\tilde{A}_{2 i-1,2 j-1}$, like all other entries in (2.8), is anti-symmetric with respect to the interchange $y_{i} \leftrightarrow y_{j}$, and thus we can drop the ordering requirement since the Pfaffian in (2.7) is then symmetric. As with (2.2), the form of (2.7) given in 27] is a summation 
corresponding to the RHS of (2.5), without the identification of the antisymmetric matrix $\tilde{A}$ nor the Pfaffian. The latter first appeared in [38, Theorem 2]. We remark too that in this reference the role of the initial condition is discussed with more precision.

If we write

$$
S(x, y ; \rho)=\frac{\rho}{2} e^{-\pi \rho^{2}(x-y)^{2} / 4}
$$

then the RHS of (2.9) can be rewritten

$$
\operatorname{sgn}\left(y_{j}-y_{i}\right)-2 \int_{y_{i}}^{y_{j}} S\left(y_{i}, u ; \rho\right) d u=: 2 I\left(y_{i}, y_{j} ; \rho\right)
$$

while

$$
\tilde{A}_{2 i, 2 j}=\left.\frac{2}{\rho^{2}} \frac{\partial}{\partial x} S(x, y ; \rho)\right|_{\substack{x=y_{i} \\ y=y_{j}}}=: \frac{2}{\rho^{2}} D\left(y_{i}, y_{j}\right) .
$$

It follows that (2.7) can be rewritten in the block form [38, Theorem 2]

$$
\rho_{(n)}^{\mathrm{c}}\left(y_{1}, \ldots, y_{n}\right)=2^{n} \operatorname{Pf}\left[\begin{array}{ll}
-I\left(\lambda_{i}, \lambda_{j} ; \rho\right) & S\left(\lambda_{i}, \lambda_{j} ; \rho\right) \\
-S\left(\lambda_{j}, \lambda_{i} ; \rho\right) & D\left(\lambda_{i}, \lambda_{j} ; \rho\right)
\end{array}\right] .
$$

Comparison with (1.5) shows

$$
\left.\rho_{(n)}^{\mathrm{c}}\left(y_{1}, \ldots, y_{n}\right)\right|_{\rho=\sqrt{2 / \pi}}=2^{n} \rho_{(n)}^{\mathrm{rG}}\left(y_{1}, \ldots, y_{n}\right),
$$

a fact that can also be read off from [38, Theorem 1 and Corollary 4]. This has the interpretation that if every particle in the $t \rightarrow \infty$ coalescence process $A+A \rightarrow A$, rescaled to have density $\rho=\sqrt{2 / \pi}$, is deleted with probability $1 / 2$ a point process identical to that of the bulk real eigenvalues of the real Ginibre ensemble results (cf. the sentence below (1.7)). Making use of (1.7) it follows that

$$
\left.E^{\mathrm{c}}(0 ; J)\right|_{\rho=\sqrt{2 / \pi}}=E^{\mathrm{rG}}(J ; 2)
$$

Recalling (2.2), and with $J=\left\{\left(x_{2 i-1}, x_{2 i}\right)\right\}$, we thus have

$$
E^{\mathrm{rG}}(J ; 2)=\operatorname{det}\left[\operatorname{sgn}\left(x_{j}-x_{i}\right) \operatorname{erfc}\left(\frac{1}{\sqrt{2}}\left|x_{j}-x_{i}\right|\right)\right],
$$

where use has been made of the classical identity $(\operatorname{Pf} A)^{2}=\operatorname{det} A$. According to the discussion below (1.7) and with $\xi$ fixed, only for $0<\xi \leq 1$ does $E^{\mathrm{rG}}(J ; \xi)$ directly relate to an observable quantity. On the other hand, it does provide a sum rule linking the quantities $\left\{E^{\mathrm{rG}}(k ; J)\right\}_{k=0,1, \ldots}$.

To fulfill our aim of obtaining the analogue of the expansions (1.3) and (1.4), we now turn our attention to a different diffusion process. 


\subsection{The annihilation process $A+A \rightarrow \emptyset$}

In the annihilation process on the line particles are freely diffusing but with the condition that colliding particles annihilate. The rescaled $t \rightarrow \infty$ limit of this system can be analyzed exactly using methods that parallel those used to analyze the coalescence process $A+A \rightarrow$ $A$. But rather than $E(0 ; J)$ admitting a structured formula like (2.2), it is the quantity $E$ (even; $J$ ) that the interval $J$ contains an even number of particles that is structured. In fact [27]

$$
E^{\mathrm{a}}(\text { even; } J)=\frac{1}{2}+\frac{1}{2} E^{\mathrm{c}}(0 ; J) \text {. }
$$

The relation (2.15) implies, via (2.6) (which remains valid with $E(0 ; J)$ replaced by $E($ even; $J)$ ), that

$$
\rho^{\mathrm{a}}\left(x_{1}, \ldots, x_{n}\right)=2^{-n} \rho^{\mathrm{c}}\left(x_{1}, \ldots, x_{n}\right),
$$

which was known to a number of authors (see [27] and references therein). Substituting in (1.5) tells us that

$$
\left.\rho^{\mathrm{a}}\left(y_{1}, \ldots, y_{n}\right)\right|_{\rho=1 / \sqrt{2 \pi}}=\rho^{\mathrm{rG}}\left(y_{1}, \ldots, y_{n}\right) .
$$

As already noted below (2.9), the reference [38] contains the explicit Pfaffian expression (2.7) and (2.11). It was observed by a referee that the latter, upon multiplication by $2^{-n}$ as required in (2.16), coincides with (1.5), (1.6). Interestingly, the follow up paper [37] shows that the dynamics of the annihilation process, with a particular infinite density initial condition, is specified by an extended Pfaffian kernel. There is also a natural dynamics for real Ginibre matrices - choose the elements to now evolve according to Dyson Brownian motion. It is not known how to fully characterise the dynamics, but the exact dynamical two-point function is known, and this is distinct from the corresponding quantity for the annihilation process [39].

An immediate consequence of (2.16), obtained by substituting (2.17) in (1.7), is that

$$
\left.E^{\mathrm{a}}(J ; \xi)\right|_{\rho=1 / \sqrt{2 \pi}}=E^{\mathrm{rG}}(J ; \xi)
$$

and thus in particular that

$$
\left.E^{\mathrm{a}}(0 ; J)\right|_{\rho=1 / \sqrt{2 \pi}}=E^{\mathrm{rG}}(0 ; J)
$$

This is significant, since $E^{\mathrm{a}}(0 ;(0, s))$ has been studied as the continuum limit of the zero temperature Glauber dynamics of the one-dimensional Ising model, and this has led to the exact result [11]

$$
\left.E^{\mathrm{a}}(0 ;(0, s))\right|_{\rho=1}=\left(\sqrt{1-\mu A_{1}(s)}-(q-1) \sqrt{-\mu A_{1}(s)}\right) e^{A_{2}(s)}
$$


where

$$
\begin{aligned}
& A_{1}(z)=\sum_{n=1}^{\infty}(-2 \mu)^{n} \int_{0}^{z} d x_{1} \cdots \int_{0}^{z} d x_{n} g\left(z, x_{1}\right) \frac{\partial}{\partial x_{1}} g\left(x_{1}, x_{2}\right) \cdots \frac{\partial}{\partial x_{1}} g\left(x_{n}, z\right) \\
& A_{2}(z)=-\frac{1}{2} \sum_{n=1}^{\infty} \frac{(-2 \mu)^{n}}{n} \int_{0}^{z} d x_{1} \cdots \int_{0}^{z} d x_{n} \frac{\partial}{\partial x_{1}} g\left(x_{1}, x_{2}\right) \cdots \frac{\partial}{\partial x_{1}} g\left(x_{n}, x_{1}\right),
\end{aligned}
$$

with

$$
g(x, y)=\frac{2}{\sqrt{\pi}} \int_{0}^{\sqrt{\pi}(y-x) q /(2(q-1))} e^{-u^{2}} d u, \quad \mu=\frac{q-1}{q^{2}}, \quad q=2 .
$$

Moreover, (2.20) has been used to compute both the small $s$ expansion

$$
\begin{aligned}
p^{\mathrm{a}}(0 ; s)= & \pi s-\pi^{2} s^{3}+\frac{\pi^{2} s^{4}}{3}+\frac{\pi^{3} s^{5}}{2}-\frac{4 \pi^{3} s^{6}}{15}-\frac{\pi^{4} s^{7}}{6}+\frac{7 \pi^{4} s^{8}}{60}+\frac{\pi^{5} s^{9}}{24} \\
& -\frac{23 \pi^{5} s^{10}}{630}-\frac{\pi^{6} s^{11}}{120}+\frac{1523 \pi^{6} s^{12}}{166320}+\frac{\pi^{6}(-64+2520 \pi) s^{13}}{1814400}+O\left(s^{14}\right)
\end{aligned}
$$

and the large $s$ expansion

$$
E^{\mathrm{a}}(0 ;(0, s))=e^{-c_{1} s+c_{2}+o(1)}
$$

respectively, where in (2.24)

$$
\begin{aligned}
& c_{1}=\frac{1}{2} \zeta(3 / 2) \approx 1.3062 \\
& c_{2}=\log 2-\frac{1}{4}+\frac{1}{4 \pi} \sum_{n=2}^{\infty} \frac{1}{n}\left(-\pi+\sum_{p=1}^{n-1} \frac{1}{\sqrt{p(n-p)}}\right) \approx 0.0627 .
\end{aligned}
$$

A crucial aspect of (2.24) is that it relies on the large $s$ expansion of (2.20) begin computed first for general $0<q<2$, and then taking the limit $q \rightarrow 2$. Hence (2.24) is not a rigorous statement, although in the paragraph including (4.1) below the leading term will be established rigorously. The necessity of such a limiting procedure is immediately apparent from the large $s$ form of the first factor in (2.20) [10]

$$
\left(\sqrt{1-\mu A_{1}(s)}-(q-1) \sqrt{-\mu A_{1}(s)}\right)=\sqrt{q(2-q)}+o(1) .
$$

The derivation of this results makes use of the relationship between $A_{1}(s)$ and the elements of a certain matrix inverse.

The derivation of the asymptotic formula for $A_{2}(s)$ makes use of the fundamental asymptotic expansion of the Wiener-Hopf determinant of an integral operator with difference kernel $K(x, y)=K(x-y, 0)$ defined on $(0, s)$ for $s \rightarrow \infty$ [25],

$$
\begin{aligned}
& \log \operatorname{det}\left(\mathbb{I}-\xi K_{(0, s)}\right) \\
& =\frac{s}{2 \pi} \int_{-\infty}^{\infty} \log (1-\xi \tilde{K}(u)) d u+\frac{1}{4 \pi^{2}} \int_{0}^{\infty} u\left|\int_{-\infty}^{\infty} e^{-i k u} \log (1-\xi \tilde{K}(k)) d k\right|^{2} d u+o(1),
\end{aligned}
$$


where $\tilde{K}(u)=\int_{-\infty}^{\infty} e^{i x u} K(x, 0) d x$. This is used in conjunction with the identity (see e.g. [14, Exercises 9.3 q.1]))

$$
\log \operatorname{det}\left(\mathbb{I}-\lambda K_{(0, s)}\right)=-\sum_{p=1}^{\infty} \frac{\lambda^{p}}{p} \int_{0}^{s} d x_{1} \cdots \int_{0}^{s} d x_{p} \prod_{l=1}^{p} K\left(x_{l}, x_{l+1}\right)
$$

where $x_{p+1}:=x_{1}$.

The sought analogue of the expansions (1.3) and (1.4) for the gap probability of the real eigenvalues in the real Ginibre ensemble is an immediate corollary of (2.19), (2.25) and (2.26).

Corollary 1. The gap probability of the real eigenvalues in the bulk of real Ginibre ensemble, which have $\rho=1 / \sqrt{2 \pi}$, has the small s expansion

$$
\begin{aligned}
& E^{\mathrm{rG}}(0 ;(0, s))=1-\frac{s}{\sqrt{2 \pi}}+\frac{s^{3}}{12 \sqrt{2 \pi}}-\frac{s^{5}}{80 \sqrt{2 \pi}}+\frac{s^{6}}{720 \pi} \\
& +\frac{s^{7}}{672 \sqrt{2 \pi}}-\frac{s^{8}}{3360 \pi}-\frac{s^{9}}{6912 \sqrt{2 \pi}}+\frac{7 s^{10}}{172800 \pi}+\frac{s^{11}}{84480 \sqrt{2 \pi}}-\frac{23 s^{12}}{5322240 \pi} \\
& -\frac{s^{13}}{1198080 \sqrt{2 \pi}}+\frac{1523 s^{14}}{3874590720 \pi}-\left(\frac{1}{762048000 \sqrt{2} \pi^{3 / 2}}+\frac{1}{19353600 \sqrt{2 \pi}}\right) s^{15}+O\left(s^{16}\right)
\end{aligned}
$$

and the large $s$ expansion

$$
E^{\mathrm{rG}}(0 ;(0, s))=e^{-\tilde{c}_{1} s+c_{2}+o(1)}
$$

where $c_{2}$ is as in (2.26) while

$$
\tilde{c}_{1}=\frac{1}{\sqrt{2 \pi}} c_{1}=\frac{1}{2 \sqrt{2 \pi}} \zeta(3 / 2)
$$

We remark that the small $s$ expansion of $p^{\mathrm{rG}}(0 ;(0, s))$ follows from (2.29) by the formula (1.10); for us it is more convenient to consider $E^{\mathrm{rG}}(0 ;(0, s))$ as this is more readily accessible numerically, as we will soon demonstrate. But before doing so, we make note of a second corollary of the exact results for the annihilation process, in particular (2.15) and (2.2) and as they apply to real Ginibre ensemble.

Corollary 2. Let $A$ be the antisymmetric matrix specified by (2.3). We have

$$
E^{\mathrm{rG}}(\text { even; } J)=\left.\operatorname{Pf} A\right|_{\rho=\sqrt{2 / \pi}}
$$

and in particular

$$
E^{\mathrm{rG}}(\text { even; }(0, s))=\frac{1}{2}+\frac{1}{2} \operatorname{erfc} \frac{s}{\sqrt{2}} \text {. }
$$




\section{Comparison with numerical computations}

We have two formulas for $E^{\mathrm{rG}}(0 ;(0, s))$ — the one implied by setting $\xi=1$ in (1.9), and the one that follows by replacing $s$ by $s / \sqrt{2 \pi}$ in (2.20). It has already been remarked that many Fredholm determinant formulas in random matrix theory are well suited to high precision numerical computation. However this requires that the kernel be analytic, whereas (2.10) shows that the entry $I(x, y)$ is not differentiable at $x=y$, and thus disallowing this approach. In relation to (2.20), according to (2.28), (2.22) can be expressed in terms of a Fredholm determinant, and furthermore the corresponding kernel is analytic. But the quantity (2.21) does not have a structure consistent with (2.28), and so again we run into difficulties.

Nonetheless, it is still possible to illustrate the validity of Corollary 1, This can be done by noting an $N / 2 \times N / 2$ (for convenience it will be assumed $N$ is even) determinant formula for $E^{\mathrm{rG}, N}(0 ;(-s, s))$ in the finite $N$ real Ginibre ensemble, and then evaluating this numerically for large $N$.

For this purpose, with $k, N$ even let $p\left(\left\{\lambda_{l}\right\}_{l=1, \ldots, k},\left\{x_{j} \pm i y_{j}\right\}_{j=1, \ldots,(N-k) / 2}\right)$ denote the probability density that there are $k$ real eigenvalues and $N-k$ complex eigenvalues at the prescribed locations. Define the generalized partition function

$$
\begin{aligned}
& Z_{N}[u, v] \\
& \quad=\sum_{\substack{k=0 \\
k \text { even }}}^{N} \int_{\mathbb{R}} d \lambda_{1} \cdots d \lambda_{k} \prod_{j=1}^{k} u\left(\lambda_{l}\right) \int_{\mathbb{R}_{+}^{2}} d x_{1} d y_{1} \cdots \int_{\mathbb{R}_{+}^{2}} d x_{(N-k) / 2} d y_{(N-k) / 2} \prod_{l=1}^{(N-k) / 2} v\left(x_{l}, y_{l}\right) .
\end{aligned}
$$

We see from this that

$$
Z_{N}\left[1-\chi_{\lambda \in(-s, s)}, 1\right]=E^{\mathrm{rG}, N}(0 ;(-s, s))
$$

where $\chi_{A}=1$ for $A$ true and $\chi_{A}=0$ otherwise. The significance of (3.1) is that the generalized partition function admits the Pfaffian form [33, 16]

$$
Z_{N}[u, v]=\frac{2^{-N(N+1) / 4}}{\prod_{l=1}^{N} \Gamma(l / 2)} \operatorname{Pf}\left[\alpha_{j, l}[u]+\beta_{j, l}[v]\right]_{j, l=1, \ldots, N},
$$

where, with $\left\{p_{j}(x)\right\}_{j=0,1, \ldots}$ monic polynomials of the labelled degree but otherwise arbitrary,

$$
\alpha_{j, l}[u]=\int_{-\infty}^{\infty} d x u(x) \int_{-\infty}^{\infty} d y u(y) e^{-\left(x^{2}+y^{2}\right) / 2} p_{j-1}(x) p_{l-1}(x) \operatorname{sgn}(y-x)
$$

(the explicit form of $\beta_{j, l}$ is also known, but this will not be required below). Moreover, if the monic polynomials are chosen as

$$
p_{2 j}(x)=x^{2 j}, \quad p_{2 j+1}(x)=x^{2 j+1}-2 j x^{2 j-1},
$$

then for $u=v=1$ and with $p<q$ the skew orthogonality

$$
\alpha_{p, q}[1]+\beta_{p, q}[1]= \begin{cases}r_{j-1}:=2 \sqrt{2 \pi} \Gamma(2 j-1), & (p, q)=(2 j-1,2 j) \\ 0, & \text { otherwise }\end{cases}
$$


holds true [16.

The above theory can be used to give the sought determinant formula for $E^{\mathrm{rG}, N}(0 ;(-s, s))$ can be derived.

Proposition 1. With $\gamma(a ; x)=\int_{0}^{x} t^{a-1} e^{-t} d t$ denoting the (lower) incomplete gamma function, we have

$$
E^{\mathrm{rG}, N}(0 ;(-s, s))=\operatorname{det}\left[\delta_{j, l}-\frac{\gamma\left(l+j-3 / 2 ; s^{2}\right)}{\sqrt{2 \pi}(\Gamma(2 j-1) \Gamma(2 l-1))^{1 / 2}}\right]_{j, l=1, \ldots, N / 2} .
$$

Proof. With the choice (3.4) it follows that for $l>j$

$$
\alpha_{j, l}[u]+\beta_{j, l}[1]=\alpha_{j, l}[u]-\alpha_{j, l}[1]+ \begin{cases}r_{(j-1) / 2}, & j \text { odd, } l=j+1 \\ 0, & \text { otherwise. }\end{cases}
$$

Furthermore, for $u(x)$ even we have $\alpha_{j, l}=0$ for $j, l$ both of the same parity. This means that every second element in the Pfaffian vanishes (i.e. there is a checkerboard pattern of zero entries - this same feature has previously been noted in relation to the computation of their being exactly $k$ real eigenvalues [1, 17, 28]). In this circumstance it is generally true that

$$
\operatorname{Pf}\left[c_{j, l}\right]_{j, l=1, \ldots, N}=\operatorname{det}\left[c_{2 j-1,2 l}\right]_{j, l=1, \ldots, N / 2},
$$

and so we have

$$
Z_{N}[u, v=1]=\frac{2^{-N(N+1) / 4}}{\prod_{l=1}^{N} \Gamma(l / 2)} \operatorname{det}\left[\delta_{j, l} r_{j-1}+\alpha_{2 j-1,2 l}[u]-\alpha_{2 j-1,2 l}[1]\right]_{j, l=1, \ldots, N / 2} .
$$

Now we set $u=1-\chi_{\lambda \in(-s, s)}$. Substituting in (3.3) we have

$$
\begin{aligned}
\alpha_{2 j-1,2 l}[u]-\alpha_{2 j-1,2 l}[1]= & \left(\left(\int_{-\infty}^{\infty}-\int_{-s}^{s}\right) d x\left(\int_{-\infty}^{\infty}-\int_{-s}^{s}\right) d y-\int_{-\infty}^{\infty} d x \int_{-\infty}^{\infty} d y\right) \\
& \times e^{-\left(x^{2}+y^{2}\right) / 2} p_{2 j-2}(x) p_{2 l-1}(y) \operatorname{sgn}(y-x) .
\end{aligned}
$$

Noting from (3.4) that

$$
p_{2 l-1}(x)=-e^{x^{2} / 2} \frac{d}{d x}\left(e^{-x^{2} / 2} p_{2 l-2}(x)\right)
$$

allows (3.8) to be simplified to read

$$
\alpha_{2 j-1,2 l}[u]-\alpha_{2 j-1,2 l}[1]=-2 \int_{-s}^{s} e^{-y^{2}} p_{2 l-2}(y) p_{2 j-2}(y) d y=-2 \gamma\left(l+j-3 / 2 ; s^{2}\right) .
$$

Substituting this result in (3.7) and performing some simple manipulations we arrive at (3.6).

We have used (3.6) to compute $E^{\mathrm{rG}, N}(0 ;(-s / 2, s / 2))$ with $N=120$, and compared it against the expansions (2.29) and (2.30). Graphical accuracy in the case of the small $s$ expansion (2.29) is found for $s<2.2$; forming $\left(\log E^{\mathrm{rG}, N}(0 ;(-s / 2, s / 2))\right) / s$ graphical accuracy is obtained in the case of the large $s$ expansion (2.30) for $s>1.3$. Thus the expansions in Corollary 1 are indeed consistent with numerical computations. 


\section{Concluding remarks}

Our approach to deducing the small and large $s$ expansions in Corollary 11 relies on the equivalence in distribution of the bulk scaling limit of the real eigenvalues for the real Ginibre ensemble and the rescaled $t \rightarrow \infty$ limit of the annihilation process $A+A \rightarrow \emptyset$. It is thus of interest to remark that certain diffusions also play a crucial role in the large distance asymptotic analysis of the gap probability for so called real Weyl random polynomials [31, 32] (see also [9]). These are random polynomials $p_{N}(z)=\sum_{n=0}^{N} a_{n} \frac{z^{n}}{\sqrt{n !}}$ where the $a_{n}$ are independent real standard Gaussians. For large $N$ the density of real zeros on the real axis is to leading order equal to $1 / \pi$. It turns out that the probability of there being no real zeros on the interval $(-s, s)$ can, for large $s$, be related to the probability that a scalar Gaussian random field, with a certain covariance, and subject to random initial conditions, at any chosen point keeps the same sign it had initially. This statement requires that the diffusion occurs in the limit of large dimension $d$, and leads to the prediction that $E(0,(-s, s))$ has the leading large $s$ form $e^{-2 \theta_{\infty} s}$ where $\theta_{\infty}$ is a certain so called persistence exponent. It's exact value is not known, although the numerical estimate $\theta_{\infty} \approx 0.41$ is quoted in [32]. Note in particular that the decay is as an exponential as in (2.30) and not a Gaussian as in the Wigner surmise (1.1). Generally an exponential form will result when the point process is compressible and thus $\int_{-\infty}^{\infty}\left(\rho_{(2)}^{T}(x, 0) / \rho+\delta(x)\right) d x>0$ [15]; for the real Ginibre eigenvalues this quantity equals $2-\sqrt{2}[16]$.

Suppose we didn't have available the small and large distance expansions (2.23) and (2.24) for the annihilation process. Could we still derive the expansions of Corollary [1]? Thus we are asking if the results of Corollary 1 can be derived using (1.9) or (1.7) and (1.5). Certainly the small distance expansion (2.29) can in principle be derived by expanding the entries (1.6) for small argument, and then substituting in (1.5) and expanding out the Pfaffian to expand the $\rho_{(n)}$ for small argument, before computing the integrals as required in (1.9) $)$. The explicit form of the joint eigenvalue probability density function $p\left(\left\{\lambda_{l}\right\}_{l=1, \ldots, k},\left\{x_{j} \pm i y_{j}\right\}_{j=1, \ldots,(N-k) / 2}\right)$ for the real and complex eigenvalues [12] tells us that for small distances $\rho_{(n)}\left(\lambda_{1}, \ldots, \lambda_{n}\right)$ is proportional to $\prod_{1 \leq j<k \leq n}\left|\lambda_{k}-\lambda_{j}\right|$ and thus for small $s \rho_{(n)}$ first contributes to (1.7) at order $s^{n(n+1) / 2}$. Therefore to obtain the expansion (2.29), which is accurate up to the term proportional to $s^{15}$, the series (1.7) can be truncated at $n=4$. An analogous approach could also be used to compute the small distance expansions of $E^{\mathrm{rG}}(k ;(0, s))$ for $k>0$, although for this to be practical $k$ itself must be small.

In relation to the large distance expansion (2.30), we require a matrix kernel analogue of (2.27). This is known (see e.g. [3, pg. 14]), but it seems that only the leading term is readily computable and simply related to the corresponding term in (2.27). Thus we have that for $s \rightarrow \infty$

$$
\log \operatorname{det}\left(\mathbb{I}_{2}-\xi K_{(0, s)}\right)=\frac{s}{2 \pi} \int_{-\infty}^{\infty} \log \operatorname{det}\left(\mathbb{I}_{2}-\xi \tilde{K}(u)\right) d u+O(1)
$$

where $\tilde{K}(u)$ refers to the component wise Fourier transform of the kernel corresponding to 
$K_{(0, s)}$. Taking the Fourier transform of the matrix elements (1.8) shows

$$
\tilde{S}(k)=e^{-k^{2} / 2}, \quad \tilde{D}(k)=i k e^{-k^{2} / 2}, \quad \tilde{I}(k)=\frac{1}{i k}\left(-1+e^{-k^{2} / 2}\right)
$$

Thus

$$
\operatorname{det}\left(\mathbb{I}_{2}-\xi \tilde{K}(u)\right)=1-\left(2 \xi-\xi^{2}\right) e^{-k^{2} / 2}
$$

Substituting in (4.1) and setting $\xi=1$ allows the integral to be evaluated, and the leading term in (2.30) is reclaimed. Moreover, this provides a rigorous derivation of (2.30) to leading order. The computation of the large distance expansion of $E^{\mathrm{rG}}(k ;(0, s))$ for $k>0$ remains an open problem (see the recent review [18] for methods used in the analogous quantity for the bulk scaled GOE).

Lastly, we will comment on a number of problems relating to the present one which require more investigation. Already noted is the task of providing high precision computation of $E^{\mathrm{rG}}(0 ;(0, s))$, or more generally $E^{\mathrm{rG}}(0 ;(0, s))$. Another is to obtain the asymptotics not of the gap probability but rather the hole probability. Thus the eigenvalues of real Ginibre matrices are in general complex, so a natural quantity is the hole probability that there are no eigenvalues in a disk of circumference $\alpha$ centred at the origin of the complex plane. In the case of the complex Ginibre ensemble (Gaussian random matrices with independent complex Gaussian entries) the large $\alpha$ asymptotics have been computed in [13] (see also [2]), and in the case of complex Weyl polynomials the leading asymptotic form is also known [34, 29]. Finally, we draw attention to the task of computing the asymptotics of the gap probability for the real eigenvalues of the real Ginibre ensemble scaled not in the bulk, but at the edge. The formula (1.9) still holds, but now the entries of the kernel (1.6) require that $S(x, y)$ be replaced by [16]

$$
S(x, y)=\frac{1}{\sqrt{2 \pi}}\left(\frac{1}{2} e^{-(x-y)^{2} / 2}\left(1-\operatorname{erf} \frac{x+y}{\sqrt{2}}\right)+\frac{e^{-y^{2}}}{2 \sqrt{2}}(1+\operatorname{erf} x)\right) .
$$

Note added: This paper was written in 2013 while the author was on long service leave, and no publication sought until the announcement of the JPhysA special issue in honour of his PhD supervisor, Professor R.J. Baxter. Very recently the result (2.24) has been used in the work of [26] on a study of the asymptotic form of probability that an $N \times N$ real Ginibre matrix has a fixed number $k$ of real eigenvalues in the limit $N \rightarrow \infty$.

\section{Acknowledgements}

Financial support for this work from the Australian Research Council is acknowledged. I thank Carlo Beenakker for sending me a copy of [4 and correspondence which pointed out the numerical studies therein of the spacing distribution for a certain ensemble of matrices related to the real Ginibre ensemble. I also thank Jonathan Edge for going to the trouble of comparing the asymptotic formula of this work against large scale simulation data extending [4], and by so doing identifying an error in the reporting of the value of $c_{2}$ in the original version of this paper. 


\section{References}

[1] G. Akemann and E. Kanzieper, Integrable structure of Ginibre's ensemble of real random matrices and a Pfaffian integration theorem, J. Stat. Phys. 129 (2007), 11591231.

[2] G. Akemann, M.J. Phillips, and L. Shifrin, Gap probabilities in non-Hermitian random matrix theory, J. Math. Phys. 50 (2009), 063504.

[3] E.L. Basor and H. Widom, Wiener-Hopf determinants with Fisher-Hartwig symbols, Operator Theory: Adv. and Appl. 147 (2004), 131-149.

[4] C.W.J. Beenakker, J.M. Edge, J.P. Dahlhaus, D.I. Pikulin, Shuo Mi, and M. Wimmer, Wigner-Poisson statistics of topological transitions in a Josephson junction, Phys. Rev. Lett. 111, 037001.

[5] D. ben Avraham, Complete exact solution of diffusion-limited coalescence $A+A \rightarrow A$, Phys. Rev. Lett. 81 (1998), 4756-4759.

[6] - On the numerical evaluation of Fredholm determinants, Math. Comp. 79 (2010), 871-915.

[7] F. Bornemann, On the numerical evaluation of distributions in random matrix theory: a review with an invitation to experimental mathematics, Markov Processes Relat. Fields 16 (2010), 803-866.

[8] A. Borodin and C.D. Sinclair, The Ginibre ensemble of real random matrices and its scaling limit, Commun. Math. Phys. 291 (2000), 177-224.

[9] A. Dembo and and S. Mukherjee, No zero-crossings for random polynomials and the heat equation, Annals Prob 43 (2015), 85-118.

[10] B. Derrida, V. Hakim, and V. Pasquier, Exact exponent for the number of persistent spins in the zero-temperature dynamics of the one-dimensional Potts model, J. Stat. Phys. 85 (1996), 763-797.

[11] B. Derrida and R. Zeitak, Distribution of domain sizes in the zero temperature Glauber dynamics of the one-dimensional Potts model, Phys. Rev. E 54 (1996), 2513-2525.

[12] A. Edelman, The probability that a random real Gaussian matrix has $k$ real eigenvalues, related distributions, and the circular law, J. Multivariate. Anal. 60 (1997), 203-232.

[13] P.J. Forrester, Some statistical properties of the eigenvalues of complex random matrices, Phys. Lett. A 169 (1992), 21-24.

[14] L Log-gases and random matrices, Princeton University Press, Princeton, NJ, 2010. 
[15] P.J. Forrester and C. Pisani, The hole probability in log-gas and random matrix ensembles, Nucl. Phys. B 374 (1992), 720-740.

[16] P.J. Forrester and T. Nagao, Eigenvalue statistics of the real Ginibre ensemble, Phys. Rev. Lett. 99 (2007), 050603.

[17] Skew orthogonal polynomials and the partly symmetric real Ginibre ensemble, J. Phys. A 41 (2008), 375003 (19pp).

[18] P.J. Forrester, Asymptotics of spacing distributions 50 years later, in 'Random matrix theory, interacting particle systems and integrable systems', MRI Publications 65, Cambridge Univ. Press, Cambridge, (2014), pp. 199-222.

[19] P.J. Forrester and N.S. Witte, Exact Wigner surmise type evaluation of the spacing distribution in the bulk of the scaled random matrix ensembles, Lett. Math. Phys. 53 (2000), 195-200.

[20] M. Gaudin, Sur la loi limite de l'espacement des valeurs propres d'une matrice aléatoire, Nucl. Phys. 25 (1961), 447-458.

[21] J. Ginibre, Statistical ensembles of complex, quaternion, and real matrices, J. Math. Phys. 6 (1965), 440-449.

[22] D.L. González and G. Téllez, Statistical behavior of domain systems, Phys. Rev. E 76 (2007), 011126.

[23] — Wigner surmise for domain systems, J. Stat. Phys. 132 (2008), 187-205.

[24] M. Jimbo, T. Miwa, Y. Môri, and M. Sato, Density matrix of an impenetrable Bose gas and the fifth Painlevé transcendent, Physica 1D (1980), 80-158.

[25] M. Kac, Toeplitz matrices, translation kernels and a related problem in probability theory, J. Stat. Phys. 21 (1954), 501-509.

[26] E. Kanzieper, M. Poplavskyi, C. Timm, R. Tribe, O. Zaboronski, What is the probability that a large random matrix has no real eigenvalues?, arXiv:1503.07926.

[27] T.O. Masser and D. ben Avraham, Correlation functions for diffusion-limited annihilation, $A+A \rightarrow$ 0, Phys. Rev. E 64 (2001), 062101.

[28] A. Mays, A geometrical triumvirate of real random matrices, Ph.D. thesis, University of Melbourne, 2012.

[29] A. Nishry, Asymptotics of the hole probability for zeros of random entire functions, IMRN 2010 (2010), 2925-2946.

[30] C.E. Porter, Statistical theories of spectra: fluctuations, Academic Press, New York, 1965. 
[31] G. Schehr and S.N. Majumdar, Statistics of the number of zero crossings: from random polynomials to the diffusion equation, Phys. Rev. Lett. 99 (2007), 060603.

[32] _ Real roots of random polynomials and zero crossing properties of diffusion equation, J. Stat. Phys. 132 (2008), 235-273.

[33] C.D. Sinclair, Averages over Ginibre's ensemble of random real matrices, Int. Math. Res. Not. 2007 (2007), rnm015.

[34] M. Sodin and B. Tsirelson, Random complex zeroes, III. Decay of the hole probability, Israel J. Math. 147 (2005), 371-379.

[35] H.-J. Sommers, Symplectic structure of the real Ginibre ensemble, J. Phys. A 40 (2007), F671-F676.

[36] T. Tao and V. Vu, Random matrices: universality of local spectral statistics of nonHermitian matrices, arXiv:1206.1893.

[37] R. Tribe, S.K. Yip, and O. Zaboronski, One-dimensional annihilating and coalescing particle systems as extended Pfaffian point processes, Elec. Commun. Probab. 17 (2012), 1-7.

[38] R. Tribe and O. Zaboronski, Pfaffian formulae for one dimensional coalescing and annihilating systems, Elec. J. Prob. 16 (2011), Article 76.

[39] _ The Ginibre evolution in the large-N limit, J. Math. Phys. 55, (2014), 063304 